\title{
Carnitine Supplementation Induces Acylcarnitine Production in Tissues of Very Long-Chain Acyl-CoA Dehydrogenase-Deficient Mice, Without Replenishing Low Free Carnitine
}

\author{
SONJA PRIMASSIN, FRANK TER VELD, ERTAN MAYATEPEK, AND UTE SPIEKERKOETTER \\ Department of General Pediatrics, University Children's Hospital, Duesseldorf D-40225, Germany
}

\begin{abstract}
Deficiency of very long-chain acyl-CoA dehydrogenase (VLCAD) results in accumulation of C14-C18 acylcarnitines and low free carnitine. Carnitine supplementation is still controversial. VLCAD knockout (VLCAD ${ }^{-1-}$ ) mice exhibit a similar clinical and biochemical phenotype to those observed in humans. $\mathrm{VLCAD}^{-1-}$ mice were fed with carnitine dissolved in drinking water. Carnitine, acylcarnitines, and $\gamma$-butyrobetaine were measured in blood and tissues. Measurements were performed under resting conditions, after exercise and after $24 \mathrm{~h}$ of regeneration. HepG2 cells were incubated with palmitoyl-CoA and palmitoyl-carnitine, respectively, to examine toxicity. With carnitine supplementation, acylcarnitine production was significantly induced. Nevertheless, carnitine was low in skeletal muscle after exercise. Without carnitine supplementation, liver carnitine significantly increased after exercise, and after $24 \mathrm{~h}$ of regeneration, carnitine concentrations in skeletal muscle completely replenished to initial values. Incubation of hepatic cells with palmitoylCoA and palmitoyl-carnitine revealed a significantly reduced cell viability after incubation with palmitoyl-carnitine. The present study demonstrates that carnitine supplementation results in significant accumulation of potentially toxic acylcarnitines in tissues. The expected prevention of low tissue carnitine was not confirmed. The principle mechanism regulating carnitine homeostasis seems to be endogenous carnitine biosynthesis, also under conditions with increased demand of carnitine such as in VLCAD-deficiency. (Pediatr Res 63: 632-637, 2008)
\end{abstract}

$\mathrm{V}_{\mathrm{A} a \mathrm{sen}}^{\mathrm{en}}$ ery long-chain acyl-CoA dehydrogenase (VLCAD or ACADVL, EC 1.3.99.3) is one of several enzymes of mitochondrial $\beta$-oxidation. Deficiency of VLCAD is the most common mitochondrial $\beta$-oxidation defect of long-chain fatty acids, with an occurrence of approximately 1:50.000 to $1: 100.000$ births (1). In humans, VLCAD-deficiency (VLCADD) is characterized by phenotypic heterogeneity. Phenotypic presentation is heterogeneous and different forms of presentation are distinguished: a severe early onset form presenting with cardiomyopathy and Reye-like symptoms; a hepatic phenotype that usually expresses in infancy with recurrent hypoketotic hypoglycemia; and a milder, later-onset, myopathic form with episodic muscle weakness and rhabdomyolysis (2). However, the hepatic phenotype of infancy will

Received October 29, 2007; accepted January 10, 2008.

Correspondence: Sonja Primassin, M.D., Department of General Pediatrics, University Children's Hospital, Moorenstraße 5, D-40225 Duesseldorf, Germany; e-mail: sonja.primassin@med.uni-duesseldorf.de

The study was financially supported by grants from the Deutsche Forschungsgemeinschaft (DFG; SP1125/1-1) and the Medical Faculty of Heinrich-Heine-University, Duesseldorf, Germany. often become a muscular phenotype during childhood and adolescence. Exercise or catabolic stress such as illnesses trigger clinical symptoms. With the start of neonatal screening programs for fatty acid oxidation defects the majority of patients are asymptomatic at time of diagnosis and remain asymptomatic with preventive measures during the first years of follow-up. Especially for this group of patients, there is a need to define risk factors for the manifestation of clinical symptoms with special respect to physical exercise. Deficient oxidation of long-chain acyl-CoAs, especially during catabolism, results in accumulation of long-chain acylcarnitines.

Carnitine is an essential metabolite in energy metabolism, because it enables long-chain acyl compounds to cross the inner mitochondrial membrane as acylcarnitines. Only inside the mitochondria, fatty acids can be metabolized by $\beta$-oxidation. The body receives carnitine from dietary sources or from endogenous synthesis. In addition, it is efficiently reabsorped by the kidney. All three factors are maintaining carnitine homoeostasis (3). $\gamma$-Butyrobetaine is the last intermediate in the carnitine biosynthesis pathway and is hydroxylated only in the liver by $\gamma$-butyrobetaine dioxygenase (EC 1.14.11.1) to yield carnitine (3). Nevertheless, $\gamma$-butyrobetaine and its presursors in the carnitine biosynthesis pathway have also been detected in different tissues like muscle, heart, and kidney. Moreover, all the enzymes forming these precursors and $\gamma$-butyrobetaine were present in these tissues except $\gamma$-butyrobetaine dioxygenase (4).

Characteristically, in VLCADD long-chain C14-C18 acylCoAs accumulate in mitochondria before the $\beta$-oxidation block. To leave the mitochondria, they are reconverted into acylcarnitine esters, which can be assayed in blood. As a result of an increased production of acylcarnitines, blood free carnitine concentrations may decrease (5). Especially physical exercise results in decreased free carnitine concentrations in skeletal muscles (6). However, earlier studies have shown that blood and tissue concentrations of free carnitine do not always correlate (6). It has been widely discussed whether supplementation of exogenous carnitine is advisable to recover intracellular carnitine concentrations $(7,8)$. In contrast, an increased supply of carnitine could result in a further increase

\footnotetext{
Abbreviations: VLCAD, very long-chain acyl-CoA dehydrogenase; VLCADD, very long-chain acyl-CoA dehydrogenase deficiency; VLCAD ${ }^{-/-}$, very longchain acyl-CoA dehydrogenase knockout
} 
of long-chain acylcarnitines, compounds associated with possibly lethal heart rhythm disturbance (9).

The VLCAD knockout $\left(\mathrm{VLCAD}^{-/-}\right)$mouse is viable and demonstrates no VLCAD-specific clinical phenotype under resting conditions in the first 6 mo of life besides an increased fat storage in tissues (10). Physical exercise or fasting, however, may induce similar clinical phenotypes to humans (11). $\mathrm{VLCAD}^{-1-}$ mice present with stress-induced hypoglycemia, skeletal myopathy, and cold intolerance associated with elevated C14-C18 acylcarnitines (6). Overall, the $\mathrm{VLCAD}^{-1-}$ mouse is an excellent model to study human VLCADD.

In the present study, we analyzed the effects of physical exercise, regeneration after physical exercise and the effect of carnitine supplementation on carnitine and acylcarnitine homeostasis in the $\mathrm{VLCAD}^{-1-}$ mouse. In addition, we analyzed $\gamma$-butyrobetaine, a carnitine biosynthesis metabolite, in tissues to define the importance of carnitine supplementation versus endogenous biosynthesis. To examine the effects of C16-CoA and C16-carnitine, respectively, on cell proliferation and viability, HepG2 cells were incubated with these metabolites.

\section{METHODS}

Concentrations of $\gamma$-butyrobetaine, carnitine, and acylcarnitines in blood and tissues were measured in VLCAD ${ }^{-1-}$ mice and wild-type (WT) littermates under well fed, resting conditions. Each group consisted of five mice aged 10-12 wk. The mean body weight of the 10-12-wk-old animals was $24.4 \pm 0.6 \mathrm{~g}$. To determine carnitine and acylcarnitines in liver, skeletal muscle, and blood in response to exercise and after $24 \mathrm{~h}$ of regeneration, mice from both genotypes $(n=5)$ were subjected to treadmill exercise. A second group of mice from both genotypes $(n=5)$ received oral carnitine supplementation for $5 \mathrm{wk}$ after weaning. Also, carnitine-supplemented mice were investigated under resting conditions, after exercise and after regeneration.

The mice were killed immediately or $24 \mathrm{~h}$ after the stress situation was terminated. Liver and skeletal muscle were removed and frozen immediately in liquid nitrogen. Blood samples were collected by heart puncture and dried on a filter paper card.

All animal studies were performed with the approval of the HeinrichHeine-University Institutional Animal Care and Use Committee. The care of the animals was in accordance with the Heinrich-Heine-University Medical Center and Institutional Animal Care and Use Committee guidelines.

Generation and genotyping of VLCAD-deficient mice. $\mathrm{VLCAD}^{-\prime-}$ mice were generated as described $(6,12)$. Genotypes were determined by duplicate PCR analyses (13).

Exercise on a treadmill. Five $\mathrm{VLCAD}^{-1-}$ and five WT mice were subjected to exercise on a treadmill equipped with an electric shock grid (10 mAmp, frequency of $10 \mathrm{~Hz}$ ). Mice had to run at a moderate speed of 16 $\mathrm{m} \cdot \mathrm{min}^{-1}$ for $60 \mathrm{~min}$. Some VLCAD ${ }^{-1-}$ mice were exhausted after $45 \mathrm{~min}$.
Exhaustion was defined as resting more than $15 \mathrm{~s} \cdot \mathrm{min}^{-1}$ on the electric shock grid or as falling back on the electric shock grid more than 15 times $\min ^{-1}$ (5).

Carnitine supplementation. $\mathrm{VLCAD}^{-1-}$ and WT mice received oral carnitine supplementation in an approximate dose of $200 \mathrm{mg} \cdot \mathrm{kg}^{-1} \cdot \mathrm{day}^{-1}$ dissolved in drinking water (L-Carn-drinking solution, $100 \mathrm{mg} \cdot \mathrm{mL}^{-1}$, sigmatau) for $5 \mathrm{wk}$. The approximate volume of drinking water was calculated at 5 $\mathrm{mL}$. The high dose of $200 \mathrm{mg} \cdot \mathrm{kg}^{-1} \cdot \mathrm{day}^{-1}$ was chosen, after supplementation of carnitine in a dose of $100 \mathrm{mg} \cdot \mathrm{kg}^{-1} \cdot \mathrm{day}^{-1}$, in accordance to clinical use, did not result in increased free carnitine concentrations in blood and tissues of $\mathrm{VLCAD}^{-1-}$ mice in previous experiments. Metabolites were measured under resting, exercised, and regenerated conditions.

Analysis of carnitine and acylcarnitines. In blood, carnitine and acylcarnitines were extracted with methanol from dried blood spots (equivalent to 25 $\mu \mathrm{L}$ of blood) and analyzed as their butyl esters using electrospray ionization tandem mass spectrometry as previously described $(14,15)$. Free carnitine (C0) and all even-chain C14-C18 acylcarnitines (saturated and unsaturated) were measured.

In tissues, analysis of $\gamma$-butyrobetaine, carnitine, and acylcarnitines was performed according to van Vlies et al. (16). In brief, liver $(\sim 60 \mathrm{mg})$ and skeletal muscle $(\sim 50 \mathrm{mg})$ pieces were lyophilized for $12 \mathrm{~h}$ including internal standards $\left(16.25 \mathrm{nmol}\left[{ }^{2} \mathrm{H}_{3}\right]\right.$ carnitine, for carnitine and $\gamma$-butyrobetaine concentrations; $0.05 \mathrm{nmol}\left[{ }^{2} \mathrm{H}_{3}\right] \mathrm{C}_{16}$-acylcarnitine, for $\mathrm{C} 14$-C18-acylcarnitines). The lyophilized tissues were powderized and dissolved in $1 \mathrm{~mL}$ of $80 \%$ acetonitrile. After homogenization and centrifugation the supernatant was dried. $\gamma$-Butyrobetaine, carnitine, and acylcarnitines were analyzed by electrospray ionization tandem mass spectrometry as their butyl esters and

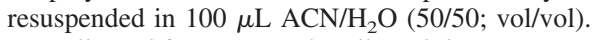

Cell proliferation and cell viability. 3-(4,5-dimethylthiazol-2-yl)-2,5diphenyltetrazolium bromide (MTT) assays were used as measure for cell proliferation and cell viability to investigate cytotoxicity of 20-120 and $100-500 \mu \mathrm{M}$ of palmitoyl-carnitine and palmitoyl-CoA, respectively, on HepG2 cells by quantifying mitochondrial dehydrogenase activity. HepG2 cells, in the log phase of growth, were plated in 96-well plates at 100,000 cells per well and after $4 \mathrm{~h}$, palmitoyl-carnitine and palmitoyl-CoA were added. Care was taken to neutralize possible inhibitory $\mathrm{pH}$ effects of the substances. After $48 \mathrm{~h}$ of incubation at $37^{\circ} \mathrm{C}$ and $5 \% \mathrm{CO}_{2}$, MTT was added to a final concentration of $250 \mu \mathrm{g} \cdot \mathrm{mL}^{-1}$ and incubated for $4 \mathrm{~h}$. Cell lysis and formazan solubilization were achieved by addition of $100 \mu \mathrm{L} 10 \%$ (wt/vol) SDS. The formazan production was determined spectrophotometrically at $550 \mathrm{~nm}$, with $670 \mathrm{~nm}$ as reference. The inhibitory effect of palmitoyl-carnitine and palmitoyl-CoA was objectified by calculating the half maximal inhibitory concentration $\left(\mathrm{IC}_{50}\right)$ by nonlinear curve fitting of a 5-parameter logistic function to the experimental data.

Statistical analyses. Data are presented as means \pm SEM with $n$ denoting the number of animals tested. Analyses for the significance of differences were performed using $t$ tests for paired and unpaired data $(p<0.05)$.

\section{RESULTS}

Free carnitine in skeletal muscle. Free carnitine concentrations were determined in skeletal muscle under resting conditions. In WT and $\mathrm{VLCAD}^{-1-}$ mice, free carnitine concentrations were comparable (Fig. 1A). We further studied the
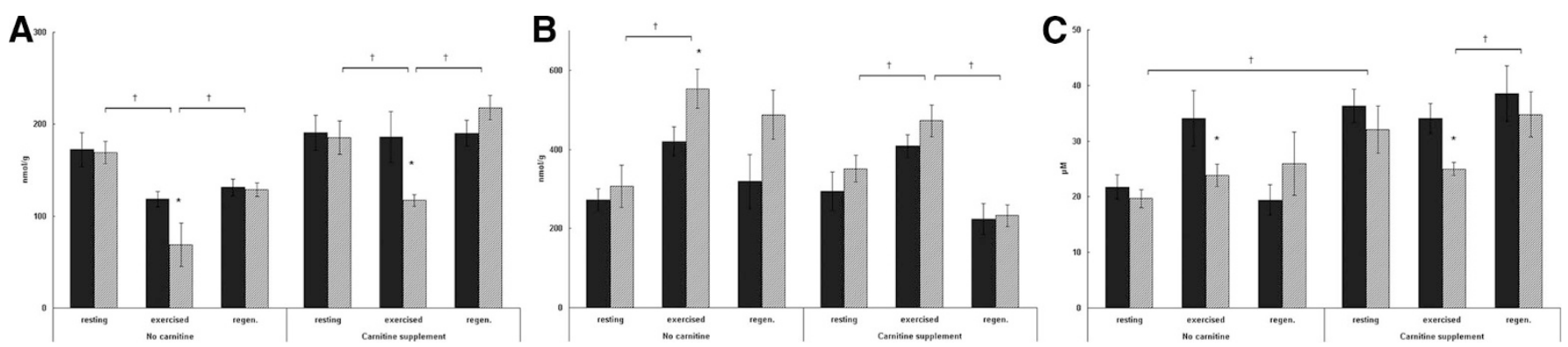

Figure 1. Free carnitine concentrations in blood and tissues. Mean free carnitine concentrations in $(A)$ muscle $\left(\mathrm{nmol} \cdot \mathrm{g}^{-1}\right.$ wet weight), $(B)$ liver $\left(\mathrm{nmol} \cdot \mathrm{g}^{-1}\right.$ wet weight), and $(C)$ blood $\left(\mu \mathrm{mol} \cdot \mathrm{L}^{-1}\right)$ under resting, exercised, and regenerated conditions are shown in $\mathrm{VLCAD}^{-\prime-}$ and WT mice and carnitine supplemented $\mathrm{VLCAD}^{-1-}$ and WT mice. Gray bars and striped bars represent WT and VLCAD ${ }^{-1-}$ mice, respectively. Values are means \pm SEM for five mice, where each tissue was analyzed in duplicate. ${ }^{*} p<0.05$ indicating significant differences between WT and $\mathrm{VLCAD}^{-/-}$mice. $\dagger p<0.05$ indicating significant differences between $\mathrm{VLCAD}^{-1-}$ mice in different groups. 
effect of exercise on free carnitine concentrations. Free carnitine decreased in muscle from WT mice and $\mathrm{VLCAD}^{-1-}$ mice. Importantly, this decrease in carnitine was significantly greater in VLCAD ${ }^{-1-}$ mice, compared with WT mice, leading to lower levels. After $24 \mathrm{~h}$ of regeneration, carnitine concentrations increased to initial levels, also in $\mathrm{VLCAD}^{-1-}$ mice. Moreover, carnitine supplementation of WT and $\mathrm{VLCAD}^{-1-}$ mice did not increase free carnitine in skeletal muscle under resting conditions, and did not prevent low carnitine concentrations after exercise in $\mathrm{VLCAD}^{-/-}$mice (Fig. 1A).

Free carnitine in liver. Concentrations were significantly increased in exercised $\mathrm{VLCAD}^{-1-}$ mice compared with resting conditions (Fig. 1B). With carnitine supplementation, exercised mice also presented with significantly higher free carnitine levels compared with resting conditions; however, concentrations were lower than without carnitine supplementation. After regeneration, free carnitine concentrations in liver tissue significantly decreased with carnitine supplementation but remained high without carnitine supplementation (Fig. 1B).

Free carnitine in blood. Carnitine supplementation resulted in higher carnitine levels under resting conditions (Fig. 1C). In WT mice without carnitine supplementation, free carnitine increased significantly after excerise; in $\mathrm{VLCAD}^{-1-}$ mice no significant changes were observed. After regeneration, free carnitine decreased again in WT mice, with respect to the exercised group. In $\mathrm{VLCAD}^{-/-}$mice, regeneration had no influence on blood carnitine. After exercise, also, carnitinesupplemented $\mathrm{VLCAD}^{-/-}$mice presented with significantly lower free carnitine; however, exercise had no effect on blood carnitine levels in WT mice. After regeneration, blood carnitine concentrations returned to initial levels in $\mathrm{VLCAD}^{-1-}$ mice (Fig. 1C).

Long-chain acylcarnitines. In skeletal muscle tissue, after physical exercise, C14-C18 acylcarnitine concentrations significantly increased in $\mathrm{VLCAD}^{-/-}$mice and decreased again after $24 \mathrm{~h}$ of regeneration (Fig. 2A). Carnitinesupplemented $\mathrm{VLCAD}^{-1-}$ mice displayed significantly higher concentrations of acylcarnitines in skeletal muscle tissue under resting conditions. Exercise resulted in a further increase of long-chain acylcarnitines in carnitinesupplemented mice. Concentrations were 4-fold higher than in resting mice without carnitine supplementation. After $24 \mathrm{~h}$ of regeneration, acylcarnitine concentrations decreased to lower levels than found under resting conditions before exercise (Fig. 2A).

In liver, acylcarnitine concentrations were elevated after exercise and decreased again in regenerated mice to levels observed at rest. Interestingly, carnitine supplementation resulted in 3-fold higher acylcarnitine concentrations in both $\mathrm{WT}$ and $\mathrm{VLCAD}^{-1-}$ resting mice, compared with mice without carnitine supplementation. In both, WT and $\mathrm{VLCAD}^{-/-}$ mice, with supplementation of carnitine, there was no change in acylcarnitine concentrations under resting, exercised or regenerated conditions (Fig. $2 B$ ).

Concentrations of C14-C18 acylcarnitines in blood of WT mice remained unchanged after exercise and regeneration. $\mathrm{VLCAD}^{-1-}$ mice displayed higher acylcarnitine levels after exercise. Carnitine supplementation resulted in increased acylcarnitines in $\mathrm{VLCAD}^{-1-}$ mice compared with WT mice (Fig. 2C).

$\gamma$-Butyrobetaine. $\gamma$-Butyrobetaine concentrations were measured as precursor of carnitine in endogenous carnitine biosynthesis. In skeletal muscle, $\gamma$-butyrobetaine concentrations significantly decreased in $\mathrm{VLCAD}^{-/-}$mice after exercise. Carnitine supplemented mice displayed, overall, much higher concentrations of $\gamma$-butyrobetaine in skeletal muscle. However, $\gamma$-butyrobetaine levels were significantly lower in muscle from $\mathrm{VLCAD}^{-1-}$ mice compared with WT littermates (Fig. 3A).

In liver, $\gamma$-butyrobetaine concentrations remained in $\mathrm{VLCAD}^{-1-}$ mice at the same level after exercise and after regeneration, although WT mice displayed significantly increased $\gamma$-butyrobetaine concentrations after exercise. Carnitine supplementation also resulted in significantly higher liver $\gamma$-butyrobetaine concentrations. Importantly, $\gamma$-butyrobetaine was significantly lower in $\mathrm{VLCAD}^{-1-}$ mice compared with WT mice after $24 \mathrm{~h}$ of regeneration (Fig. 3B).

Cell proliferation and cell viability. Proliferation and viability of HepG2 cells is significantly affected after incubation with C16:0-carnitine in the MTT-assay. The $\mathrm{IC}_{50}$ for cells with C16:0-CoA was $337 \mu \mathrm{M}$. Cells with C16:0-carnitine had an $\mathrm{IC}_{50}$ of $76 \mu \mathrm{M}$ (Fig. 4). So the cytotoxicity of C16:0carnitine is 4-fold higher than the cytotoxicity of C16:0-CoA.
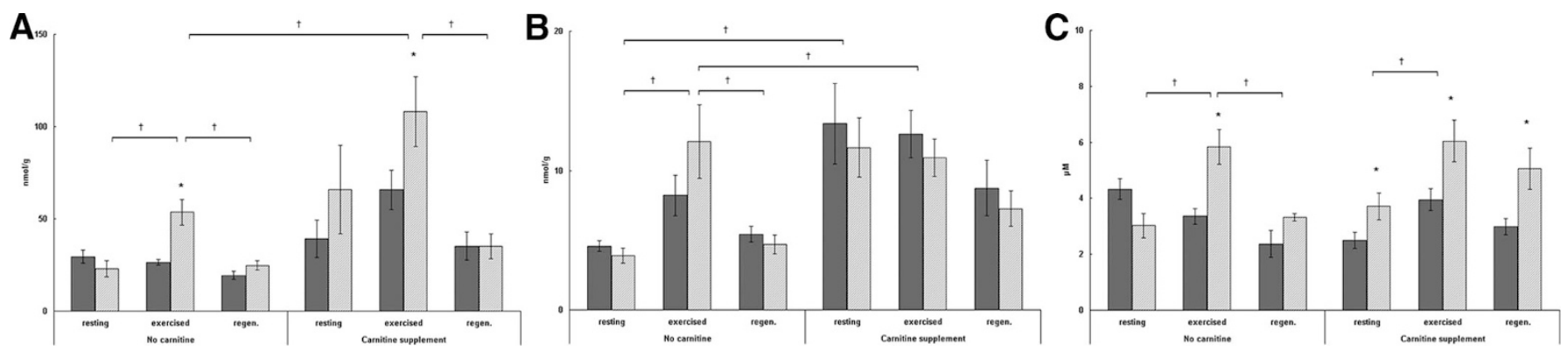

Figure 2. Acylcarnitine concentrations in blood and tissues. Mean acylcarnitine concentrations in $(A)$ muscle $\left(\mathrm{nmol}^{-1} \mathrm{~g}^{-1}\right.$ wet weight), $(B)$ liver $\left(\mathrm{nmol} \cdot \mathrm{g}^{-1}\right.$ wet weight), and $(C)$ blood $\left(\mu \mathrm{mol} \cdot \mathrm{L}^{-1}\right)$ under resting, exercised, and regenerated conditions are shown in $\mathrm{VLCAD}^{-\prime-}$ and WT mice and carnitine supplemented $\mathrm{VLCAD}^{-1-}$ and WT mice. Gray bars and striped bars represent WT and $\mathrm{VLCAD}^{-1-}$ mice, respectively. Values are means \pm SEM for five mice, where each tissue was analyzed in duplicate. ${ }^{*} p<0.05$ indicating significant differences between WT and $\mathrm{VLCAD}^{-1-}$ mice. $\dagger p<0.05$ indicating significant differences between $\mathrm{VLCAD}^{-\prime-}$ mice in different groups. 

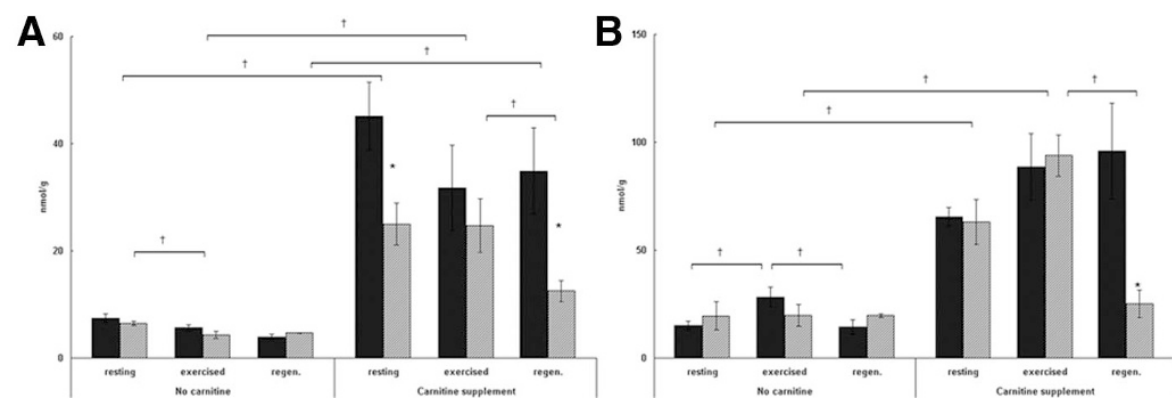

Figure 3. $\gamma$-Butyrobetaine concentrations in muscle and liver tissue. Mean $\gamma$-butyrobetaine concentrations in $(A)$ muscle $\left(\mathrm{nmol} \cdot \mathrm{g}^{-1}\right.$ wet weight) and $(B)$ liver $\left(\mathrm{nmol} \cdot \mathrm{g}^{-1}\right.$ wet weight) under resting, exercised, and regenerated conditions are shown in $\mathrm{VLCAD}^{-1-}$ and WT mice and carnitine supplemented VLCAD ${ }^{-1-}$ and WT mice. Gray bars and striped bars represent WT and $\mathrm{VLCAD}^{-1-}$ mice, respectively. Values are means \pm SEM for five mice, where each tissue was analyzed in duplicate. ${ }^{*} p<0.05$ indicating significant differences between WT and $\mathrm{VLCAD}^{-1-}$ mice. $\dagger p<0.05$ indicating significant differences between $\mathrm{VLCAD}^{-1-}$ mice in different groups.

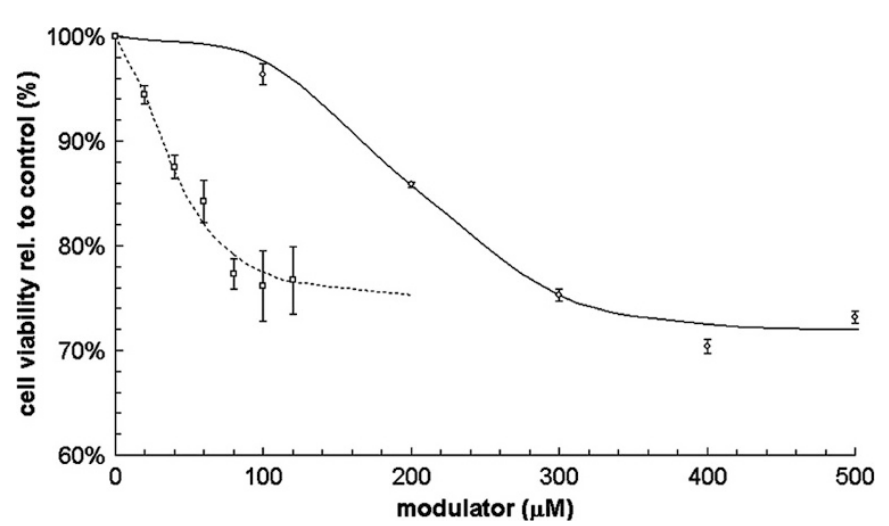

Figure 4. Cell proliferation and cell viability. Response of HepG2 cells to palmitoyl-carnitine and palmitoyl-CoA revealed by MTT assay. HepG2 cells were plated and incubated $4 \mathrm{~h}$ later with $\mathrm{C} 16: 0-\mathrm{CoA}(\leq)$ or C16:0-carnitine, respectively, for $48 \mathrm{~h}$. Curves represent nonlinear curve fitting of a 5-parameter logistic function to the experimental data.

\section{DISCUSSION}

In the present study, we demonstrated the effect of carnitine supplementation on exercise-induced changes in carnitine and acylcarnitine concentrations in skeletal muscle, liver, and blood from WT and VLCAD ${ }^{-1-}$ mice. The dose of supplemented carnitine was chosen double compared with usual clinical use as previous experiments in $\mathrm{VLCAD}^{-/-}$mice showed that low carnitine levels after exercise were not prevented with the usual dose of $100 \mathrm{mg} \cdot \mathrm{kg}^{-1} \cdot \mathrm{day}^{-1}$ (17). In addition to the metabolic steps involved in the consumption of carnitine, we also analyzed $\gamma$-butyrobetaine in both murine skeletal muscle and liver to gain more insight into the intrinsic pathways of carnitine biosynthesis.

Carnitine supplementation and acylcarnitine concentrations. Our current study in the murine model of VLCAD demonstrates for the first time a significant rise in acylcarnitines observed in skeletal muscle when mice are supplemented with carnitine (17). With exercise and an increase in acyl-CoA, the production of acylcarnitines after carnitine supplementation is even greater compared with animals without carnitine supplementation. This has previously been assumed, but it has never been shown at tissue level. Indication for carnitine supplementation in disorders with secondary carnitine deficiency such as VLCADD has been 1) increased urinary elimination of accumulating acyl-CoAs as acylcarnitines and 2) provision of sufficient intramitochondrial CoA for other metabolic pathways. The significant production of acylcarnitines after carnitine supplementation was observed irrespective of the studied genotype and questions the beneficial role of carnitine supplementation in patients, although being based on a mouse-model. Of note, it is well documented that both acylcarnitines and acyl-CoAs are potentially toxic for the cells as they may be inhibiting other important pathways in the cells $(18,19)$. Because of the increase of acylcarnitines induced by carnitine supplementation is more pronounced in $\mathrm{VLCAD}^{-1-}$ mouse muscle, the overall result of exercise in combination with carnitine supplementation is a near 5-fold increase in acylcarnitine levels in $\mathrm{VLCAD}^{-/-}$ mouse muscle compared with muscle from nonsupplemented WT mice under resting conditions.

Similar to muscle from carnitine-supplemented mice, liver acylcarnitine levels are increased on carnitine supplementation, albeit not as much as in muscle. Because liver oxidative capacity is far lower than in skeletal muscle, concentrations of acylcarnitines in liver tissue are overall lower than in skeletal muscle. Our studies on the cytotoxic effects of palmitoylcarnitine and palmitoyl-CoA in proliferating hepatic cells revealed approximately 4-fold higher cytotoxicity of palmitoylcarnitine, illustrating the negative effect of acylcarnitine accumulation in liver cells (Fig. 4).

Free carnitine and acylcarnitine dynamics in blood and skeletal muscle. As expected, carnitine supplementation results in a near 2-fold increase in blood carnitine concentration in resting WT and $\mathrm{VLCAD}^{-1-}$ mice. After exercise, free carnitine in blood is significantly lower in $\mathrm{VLCAD}^{-1-}$ mice compared with WT mice, irrespective of carnitine supplementation. These low blood concentrations reflect the decreased levels in $\mathrm{VLCAD}^{-1-}$ mouse muscle and suggest impaired carnitine handling under exercised conditions with and without supplementation of carnitine. Supplemented carnitine, therefore, fails to prevent the significant drop in muscle free carnitine under exercised conditions. But, even without supplemented carnitine, free carnitine levels replenish again within $24 \mathrm{~h}$ of regeneration. Furthermore, carnitine supplementation does not prevent increased blood acylcarnitine levels after exercise. Acylcarnitines are high in blood as soon as there are increased concentrations in any organ, because they 
are thought to leave the cells and go into the circulation to finally be excreted via the urine from the body $(20,21)$. Overall, based on our current findings, using the murine model of VLCAD, carnitine supplementation does not prevent low carnitine concentrations in muscle after exercise and moreover, supplementation actually results in a sharp rise of acylcarnitines in skeletal muscle.

Liver carnitine biosynthesis is no longer coupled to muscle carnitine demand during carnitine supplementation. As a marker for carnitine biosynthesis, we studied $\gamma$-butyrobetaine levels in murine liver and skeletal muscle and found that carnitine supplementation greatly influences the dynamics of this precursor. First, we observed the profound effect of exercise stress on $\gamma$-butyrobetaine in skeletal muscle in $\mathrm{VLCAD}^{-1-}$ mice, with considerably lower $\gamma$-butyrobetaine concentrations. As skeletal muscle does not possess the full machinery to biosynthesize carnitine, muscle tissue lacks $\gamma$-butyrobetaine dioxygenase (4), we assume that the observed decrease in $\gamma$-butyrobetaine after exercise in $\mathrm{VLCAD}^{-1-}$ mice represents transport of $\gamma$-butyrobetaine out of the muscle cell to supply precursor for the biosynthesis of carnitine in liver. Also, Vaz and Wanders (3) postulated that $\gamma$-butyrobetaine is produced in different kinds of tissues and then excreted into the circulation to be transported to a tissue that contains $\gamma$-butyrobetaine dioxygenase. Overall, after carnitine supplementation $\gamma$-butyrobetaine concentrations are significantly higher irrespective of the genotype suggesting decreased demand of carnitine biosynthesis. However, $\mathrm{VLCAD}^{-1-}$ mice presented with significantly lower $\gamma$-butyrobetaine concentrations in muscle than WT mice and, after $24 \mathrm{~h}$ of regeneration, $\gamma$-butyrobetaine was significantly decreased compared with resting conditions. These findings might implicate that in all nonsupplemented animals and in supplemented $\mathrm{VLCAD}^{-/-}$ mice, there is still a higher demand for carnitine biosynthesis, especially during exercise stress because liver carnitine production is augmented (Fig. 2B). The observed increase in liver carnitine concentration during exercise, with and without carnitine supplementation, is indicative of $\gamma$-butyrobetaine being converted into carnitine by $\gamma$-butyrobetaine dioxygenase rather than liver $\gamma$-butyrobetaine production being reduced. This would be in-line with the observed decrease in $\gamma$-butyrobetaine in liver after exercise or within $24 \mathrm{~h}$ of regeneration. Importantly, after $24 \mathrm{~h}$ of regeneration, liver carnitine levels remain high in nonsupplemented $\mathrm{VLCAD}^{-/-}$mice, indicating an increased and sustained demand for carnitine in this mouse phenotype during the recovery phase. In $\mathrm{VLCAD}^{-1-}$ mice receiving oral supplementation of carnitine, liver carnitine returns to basic levels after $24 \mathrm{~h}$ of regeneration reflecting that high blood carnitine in carnitine supplemented $\mathrm{VLCAD}^{-1-}$ animals suppresses carnitine biosynthesis in liver, at least to some degree. Whether the gene for $\gamma$-butyrobetaine dioxygenase is up-regulated in $\mathrm{VLCAD}^{-1-}$ mice or up-regulated after regular exercise in response to the secondary carnitine deficiency is currently under investigation.

Blood carnitine and acylcarnitine profiling does not fully mirror cellular carnitine metabolism. Within the framework of clinical diagnostics, acylcarnitine profiling of dried bloodspots represents a powerful tool for clinicians to gain insight into fatty acid metabolism. However, blood acylcarnitine profiles can merely represent an overall response of all organs involved in carnitine metabolism. The consequence of this is that blood acylcarnitines do not necessarily reflect concentration changes taking place in individual organ systems (6); thus, even a moderate increase in blood acylcarnitines should be treated with caution. For example, we observed increased acylcarnitine levels in skeletal muscle after oral supplementation of carnitine whereas blood acylcarnitine levels remained nearly unchanged. Only under stressed conditions, blood acylcarnitines actually reflect acylcarnitine dynamics in skeletal muscle, but not at rest.

In conclusion, in the murine model of VLCAD we observed a significant increase in acylcarnitine production after oral supplementation of carnitine in skeletal muscle, especially after exercise. However, carnitine supplementation does not prevent decreased free carnitine in muscle after exercise. Furthermore, exercise triggers carnitine biosynthesis with replenishment of low free carnitine pools in muscle within $24 \mathrm{~h}$. Therefore, if we extrapolate our findings obtained after $1 \mathrm{~h}$ of moderate stress in this mouse model, it may well be that carnitine supplementation is not at all beneficial for affected patients with VLCADD. More importantly, we rather have to consider that carnitine supplementation may actually be a risk factor because carnitine supplementation results in significant accumulation of toxic acylcarnitines in mice tissues.

Acknowledgments. We thank Dr. M. Laryea and G. Schmitz for technical assistance.

\section{REFERENCES}

1. Spiekerkoetter U, Sun B, Zytkovicz T, Wanders R, Strauss AW, Wendel U 2003 MS/MS-based newborn and family screening detects asymptomatic patients with very-long-chain acyl-CoA dehydrogenase deficiency. J Pediatr 143:335-342

2. Gregersen N, Bross P, Andresen BS 2004 Genetic defects in fatty acid betaoxidation and acyl-CoA dehydrogenases. Molecular pathogenesis and genotypephenotype relationships. Eur J Biochem 271:470-482

3. Vaz FM, Wanders RJ 2002 Carnitine biosynthesis in mammals. Biochem J 361:417429

4. van Vlies N, Wanders RJ, Vaz FM 2006 Measurement of carnitine biosynthesis enzyme activities by tandem mass spectrometry: differences between the mouse and the rat. Anal Biochem 354:132-139

5. Spiekerkoetter U, Tokunaga C, Wendel U, Mayatepek E, Exil V, Duran M, Wijburg FA, Wanders RJ, Strauss AW 2004 Changes in blood carnitine and acylcarnitine profiles of very long-chain acyl-CoA dehydrogenase-deficient mice subjected to stress. Eur J Clin Invest 34:191-196

6. Spiekerkoetter U, Tokunaga C, Wendel U, Mayatepek E, Ijlst L, Vaz FM, van Vlies N, Overmars H, Duran M, Wijburg FA, Wanders RJ, Strauss AW 2005 Tissue carnitine homeostasis in very-long-chain acyl-CoA dehydrogenase-deficient mice. Pediatr Res 57:760-764

7. Spiekerkotter U, Schwahn B, Korall H, Trefz FK, Andresen BS, Wendel U 2000 Very-long-chain acyl-coenzyme A dehydrogenase (VLCAD) deficiency: monitoring of treatment by carnitine/acylcarnitine analysis in blood spots. Acta Paediatr 89:492495

8. Touma EH, Rashed MS, Vianey-Saban C, Sakr A, Divry P, Gregersen N, Andresen BS 2001 A severe genotype with favourable outcome in very long chain acyl-CoA dehydrogenase deficiency. Arch Dis Child 84:58-60

9. Bonnet D, Martin D, Pascale DL, Villain E, Jouvet P, Rabier D, Brivet M, Saudubray JM 1999 Arrhythmias and conduction defects as presenting symptoms of fatty acid oxidation disorders in children. Circulation 100:2248-2253

10. Exil VJ, Gardner CD, Rottman JN, Sims H, Bartelds B, Khuchua Z, Sindhal R, Ni G, Strauss AW 2006 Abnormal mitochondrial bioenergetics and heart rate dysfunction in mice lacking very-long-chain acyl-CoA dehydrogenase. Am J Physiol Heart Circ Physiol 290:H1289-H1297

11. Cox KB, Hamm DA, Millington DS, Matern D, Vockley J, Rinaldo P, Pinkert CA, Rhead WJ, Lindsey JR, Wood PA 2001 Gestational, pathologic and biochemical differences between very long-chain acyl-CoA dehydrogenase deficiency and longchain acyl-CoA dehydrogenase deficiency in the mouse. Hum Mol Genet 10:20692077 
12. Papaioannou VE, Johnson RS 1993 Gene Targeting: A Practical Approach. Oxford University Press, Oxford, pp 107-146

13. Exil VJ, Roberts RL, Sims H, McLaughlin JE, Malkin RA, Gardner CD, Ni G, Rottman JN, Strauss AW 2003 Very-long-chain acyl-coenzyme a dehydrogenase deficiency in mice. Circ Res 93:448-455

14. Chace DH, DiPerna JC, Mitchell BL, Sgroi B, Hofman LF, Naylor EW 2001 Electrospray tandem mass spectrometry for analysis of acylcarnitines in dried postmortem blood specimens collected at autopsy from infants with unexplained cause of death. Clin Chem 47:1166-1182

15. Vreken P, van Lint AE, Bootsma AH, Overmars H, Wanders RJ, van Gennip AH 1999 Quantitative plasma acylcarnitine analysis using electrospray tandem mass spectrometry for the diagnosis of organic acidaemias and fatty acid oxidation defects. J Inherit Metab Dis 22:302-306

16. van Vlies N, Tian L, Overmars H, Bootsma AH, Kulik W, Wanders RJ, Wood PA, Vaz FM 2005 Characterization of carnitine and fatty acid metabolism in the long-chain acyl-CoA dehydrogenase-deficient mouse. Biochem J 387:185-193
17. Liebig M, Gyenes M, Brauers G, Ruiter JP, Wendel U, Mayatepek E, Strauss AW, Wanders RJ, Spiekerkoetter U 2006 Carnitine supplementation induces long-chain acylcarnitine production-studies in the VLCAD-deficient mouse. J Inherit Metab Dis 29:343-344

18. Adams RJ, Cohen DW, Gupte S, Johnson JD, Wallick ET, Wang T, Schwartz A 1979 In vitro effects of palmitylcarnitine on cardiac plasma membrane $\mathrm{Na}, \mathrm{K}$ ATPase, and sarcoplasmic reticulum $\mathrm{Ca} 2+$-ATPase and $\mathrm{Ca} 2+$ transport. J Biol Chem 254:12404-12410

19. Ciapaite J, Bakker SJ, Diamant M, van Eikenhorst G, Heine RJ, Westerhoff HV, Krab K 2006 Metabolic control of mitochondrial properties by adenine nucleotide translocator determines palmitoyl-CoA effects. Implications for a mechanism linking obesity and type 2 diabetes. FEBS J 273:5288-5302

20. Bennett MJ, Rinaldo P, Strauss AW 2000 Inborn errors of mitochondrial fatty acid oxidation. Crit Rev Clin Lab Sci 37:1-44

21. Rinaldo P 2001 Fatty acid transport and mitochondrial oxidation disorders. Semin Liver Dis 21:489-500 